\title{
16S rRNA Gene Sequence of Neorickettsia helminthoeca and Its Phylogenetic Alignment with Members of the Genus Ehrlichia
}

\author{
CHARLES PRETZMAN, ${ }^{1}$ DAVID RALPH, ${ }^{2}$ DIANE R. STOTHARD,${ }^{1}$ PAUL A. FUERST, ${ }^{1}$ \\ AND YASUKO RIKIHISA ${ }^{3 *}$ \\ Department of Molecular Genetics ${ }^{1}$ and Department of Veterinary Pathobiology, ${ }^{3}$ The Ohio State University, \\ Columbus, Ohio 43210, and The California Institute of Biological Research, La Jolla, California $92037^{2}$
}

\begin{abstract}
Neorickettsia helminthoeca (tribe Ehrlichieae, family Rickettsiaceae) is the agent of salmon poisoning disease, which affects members of the family Canidae. This bacterium is unusual in that it is the only known obligately intracellular bacterium that is transmitted via a helminth vector. The nucleotide sequence of the $N$. helminthoeca 16S rRNA gene was determined and compared with the sequences of intracellular bacteria belonging to the alpha subgroup of the Proteobacteria. The $N$. helminthoeca sequence was most similar to the sequences of two Ehrlichia species, Ehrlichia risticii and Ehrlichia sennetsu (levels of sequence similarity, >95\%). All other members of the tribe Ehrlichieae, including members of the other Ehrlichia species, and the related species Cowdria ruminantium and Anaplasma marginale, were only distantly related phylogenetically (levels of sequence similarity, 84 to $86 \%$ ). Our results corroborate the results of previous ultrastructural and Western blot (immunoblot) comparisons of $N$. helminthoeca with other ehrlichial species. The genus Ehrlichia is phylogenetically incoherent and can be separated into three identifiable clusters of species. Each cluster is closely associated with a species classified in another non-Ehrlichia bacterial genus. The close relationships among $N$. helminthoeca, $E$. risticii, and $E$. sennetsu and the striking differences between these organisms and other members of the tribe Ehrlichieae suggest that in the future, these organisms should be treated as members of a new bacterial genus separate from the genus Ehrlichia.
\end{abstract}

Neorickettsia helminthoeca is the first and only obligately helminth-borne pathogenic bacterium that has been recognized (11). This obligately intracellular bacterium infects the fluke Nanophyetus salmincola, which in turn infects salmonid fish. $N$. helminthoeca can cause a severe disease in dogs or wild members of the family Canidae, referred to as salmon poisoning disease, if an animal ingests salmonid tissue that is infested with the infected trematode $(7,12)$. The disease is indigenous around rivers of the Pacific coast of the United States from northern California to southwestern Washington (3). Adult trematodes can live in the intestinal lumen of a canine host and shed infected eggs into the environment via the feces of the animal. The trematode requires another specific intermediate host, the snail Oxytrema silicula, for completion of its life cycle. $N$. helminthoeca is maintained by transovarial passage in the helminth and is found throughout the life cycle of the fluke, including in free-swimming cercariae $(9,10)$. Salmon poisoning disease is a pathological consequence of infection by $N$. helminthoeca, not the fluke or other salmon properties. The disease is acute and is characterized by fever, depression, anorexia, dehydration, diarrhea, and severe lymphadenopathy. The fatality rate in untreated infected dogs approaches $90 \%$ (3, 12). $N$. helminthoeca has been successfully isolated in a dog continuous cell line (17). This allows workers to cultivate and obtain the organism in sufficient quantities for antigenic and genetic analyses.

On the basis of the results of morphological comparisons (17) and, more recently, antigenic cross-reactivity data (14), it has been suggested that $N$. helminthoeca may be very closely related to some organisms currently placed in the genus $E h r$ lichia (tribe Ehrlichieae, family Rickettsiaceae) (15). Morpho-

${ }^{*}$ Corresponding author. Mailing address: Department of Veterinary Pathobiology, College of Veterinary Medicine, The Ohio State University, 1925 Coffey Rd., Columbus, OH 43210-1093. Phone: (614) 292-9677. Fax: (614) 292-6473. logically, N. helminthoeca and two Ehrlichia species, Ehrlichia risticii (the agent of Potomac horse fever) and Ehrlichia sennetsu (the agent of human Sennetsu fever), are very similar (17). As determined by antigenic cross-reactivity between $N$. helminthoeca and various Ehrlichia species, $N$. helminthoeca is antigenically more closely related to Ehrlichia sennetsu and Ehrlichia risticii than either it or the last two species are related to Ehrlichia canis (14). Previously, live N. helminthoeca cells were not maintained in any laboratory, and cultivation of large numbers of organisms was not possible. Consequently, phylogenetic comparisons of $N$. helminthoeca with other members of the Rickettsiales in which nucleic acid sequences were used were not possible previously. In this paper we report the results of a comparative study of the 16S rRNA gene sequences of $N$. helminthoeca, related members of the genus Ehrlichia, and other intracellular bacteria belonging to the alpha subgroup of the Proteobacteria.

\section{MATERIALS AND METHODS}

Culture of $N$. helminthoeca. $N$. helminthoeca which was previously isolated and cultured in dog macrophage cell line DH82 (17) was propagated in Dulbecco's modified minimal essential medium (GIBCO, Grand Island, N.Y.) containing $10 \%$ fetal bovine serum and $2 \mathrm{mM}$ L-glutamine in $5 \% \mathrm{CO}_{2}-95 \%$ air (17). Cells were harvested when the infection rate was $90 \%$, as determined by microscopic examination of cytocentrifuged cells stained with Diff-Quik (Baxter Scientific Products, Obetz, Ohio).

Infected cell suspensions were pooled and centrifuged at $10,000 \times g$ for $10 \mathrm{~min}$ at $4^{\circ} \mathrm{C}$, and the supernatant was discarded. The pellet was suspended in Dulbeco's modified minimal essential medium at a concentration of $5 \times 10^{6}$ cells per $\mathrm{ml}$, and the suspension was sonically disrupted at $20 \mathrm{kHz}$ for $5 \mathrm{~min}$. Unbroken cells and nuclei were pelleted by centrifugation and resuspended in Dulbecco's modified minimal essential medium. The sonic lysis procedure was repeated twice, and the supernatants were reserved, pooled, and kept at $4^{\circ} \mathrm{C}$.

DNA extraction. $N$. helminthoeca was purified by passing supernatants from sonic lysates through Sephacryl S-1000 (Pharmacia, Uppsala, Sweden) low-pressure liquid chromatography columns as previously described (13). Purified organisms were washed and pelleted from sterile phosphate-buffered saline $(9 \mathrm{mM}$ $\mathrm{Na}_{2} \mathrm{HPO}_{4}, 6 \mathrm{mM} \mathrm{NaH} \mathrm{PO}_{4}, 150 \mathrm{mM} \mathrm{NaCl} ; \mathrm{pH} 7.4$ ). After suspension in TE buffer (40 mM Tris, $1 \mathrm{mM}$ EDTA; $\mathrm{pH} 8.0$ ) containing $1 \%$ sodium dodecyl sulfate and $20 \mu \mathrm{g}$ of proteinase K (Sigma Chemical Co., St. Louis, Mo.) per ml, the 
TABLE 1. Levels of similarity and evolutionary distances between 16S rRNA gene sequences

\begin{tabular}{|c|c|c|c|c|c|c|c|c|c|c|}
\hline \multirow[b]{2}{*}{ Organism } & \multicolumn{10}{|c|}{ Level of similarity or evolutionary distance ${ }^{a}$} \\
\hline & 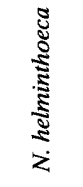 & 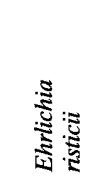 & 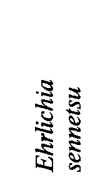 & 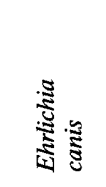 & 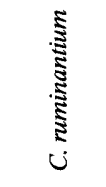 & 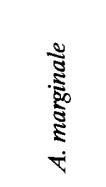 & 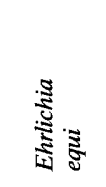 & 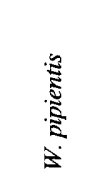 & 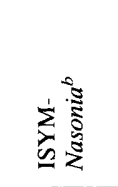 & 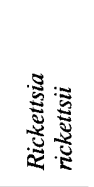 \\
\hline N. helminthoeca & & 0.0409 & 0.0412 & 0.1552 & 0.1522 & 0.1588 & 0.1561 & 0.1664 & 0.1696 & 0.1769 \\
\hline Ehrlichia risticii & 96.05 & & 0.0065 & 0.1696 & 0.1659 & 0.1625 & 0.1592 & 0.1689 & 0.1748 & 0.1847 \\
\hline Ehrlichia sennetsu & 96.03 & 99.36 & & 0.1657 & 0.1619 & 0.1603 & 0.1572 & 0.1657 & 0.1718 & 0.1827 \\
\hline Ehrlichia canis & 86.17 & 85.09 & 85.38 & & 0.0241 & 0.0748 & 0.0770 & 0.1330 & 0.1357 & 0.1802 \\
\hline C. ruminantium & 86.43 & 85.36 & 85.67 & 97.64 & & 0.0722 & 0.0722 & 0.1263 & 0.1331 & 0.1681 \\
\hline A. marginale & 85.93 & 85.63 & 85.81 & 92.98 & 93.22 & & 0.0264 & 0.1367 & 0.1401 & 0.1731 \\
\hline Ehrlichia equi & 86.12 & 85.89 & 86.03 & 92.78 & 93.21 & 87.42 & & 0.1295 & 0.1315 & 0.1760 \\
\hline W. pipientis & 85.33 & 85.18 & 85.42 & 88.07 & 88.62 & 87.76 & 88.31 & & 0.0086 & 0.1747 \\
\hline ISYM-Nasonia & 85.08 & 84.73 & 84.94 & 87.84 & 88.17 & 87.48 & 88.14 & 99.15 & & 0.1835 \\
\hline Rickettsia rickettsii & 84.40 & 83.83 & 83.98 & 84.21 & 85.12 & 84.76 & 84.52 & 84.66 & 84.02 & \\
\hline
\end{tabular}

${ }^{a}$ The values on the upper right are the average numbers of substitutions per sequence position (evolutionary distances), adjusted as described by the Kimura two-parameter model for multiple substitutions at individual positions and calculated for 1,446 positions which could be aligned unambiguously. The values on the lower left are the levels of fractional nucleotide identity between sequences.

${ }^{b}$ ISYM-Nasonia, incompatability endosymbiont of Nasonia sp.

lysate was incubated at $65^{\circ} \mathrm{C}$ for $2 \mathrm{~h}$. After extraction with phenol-chloroform and chloroform, DNA was precipitated from the aqueous phase by adding 3 volumes of cold $95 \%$ ethanol in the presence of $0.3 \mathrm{M}$ sodium acetate $(\mathrm{pH} \mathrm{5.2)}$, and the mixture was kept at $-70^{\circ} \mathrm{C}$ for $20 \mathrm{~min}$. The precipitated DNA was collected by centrifugation, and the pellet was washed once with $70 \%$ ethanol, dried, and redissolved in sterile distilled water. The DNA was examined spectrophotometrically at $A_{260}$ and $A_{280}$ and stored as $50-\mu \mathrm{g}$ aliquots at $-20^{\circ} \mathrm{C}$ until it was used.

PCR amplification of the 16S rRNA gene. PCR was used to amplify the 16S rRNA gene from DNA extracts of $N$. helminthoeca. To do this, we used two primers that included $B g l I I$ and SalI restriction sites to facilitate cloning into compatible vectors. We used the following two primers, which flanked the coding region of the 16S rRNA gene and originally were designed to amplify the entire 16S rRNA gene in Rickettsia spp. (20): RA-17 (5'-GGCTGCAGTCGACGTT TGATCCTGGCTCAG-3') and R3-17 (5'-CCAGATCTGAGCTCAAGGAG GTGATCCAGCC-3'). PCR was performed for 35 cycles (each cycle consisted of $1.5 \mathrm{~min}$ at $94^{\circ} \mathrm{C}, 3 \mathrm{~min}$ at $40^{\circ} \mathrm{C}$, and $3 \mathrm{~min}$ of extension at $70^{\circ} \mathrm{C}$ ) with an Ericomp thermocycler (Ericomp, San Diego, Calif.). Each reaction mixture contained 1 $\mu \mathrm{g}$ of template DNA, each primer at a concentration of $50 \mathrm{pM}$, each deoxynucleoside triphosphate at a concentration of $5 \mathrm{mM}$, and $2.5 \mathrm{U}$ of $\mathrm{Taq}$ polymerase (U.S. Biochemicals, Cleveland, Ohio) in buffer supplied by the manufacturer. The reaction mixtures were overlaid with $100-\mu 1$ portions of sterile mineral oil (Sigma). The mineral oil overlay was removed by chloroform extraction, and 0.1 volume of the reaction mixture was examined visually on $1.5 \%$ agarose gels stained with ethidium bromide. A single amplified product that was approximately $1.5 \mathrm{~kb}$ long was observed. The reaction products were extracted with phenol-chloroform and chloroform and precipitated in $70 \%$ ethanol at $-70^{\circ} \mathrm{C}$ for $30 \mathrm{~min}$. The precipitates were collected by centrifugation, washed once with $70 \%$ ethanol, and dissolved in a minimum volume of distilled water. The PCR product was purified by using Geneclean (BIO 101 Inc., La Jolla, Calif.), and the resulting product was suspended in TE buffer.

Cloning and sequencing the $16 S$ rRNA gene. The PCR product was digested with restriction enzymes $B g l \mathrm{II}$ and $S a l I$ according to the instructions of the manufacturer (Boehringer Mannheim, Indianapolis, Ind.). The digests were extracted with phenol-chloroform and precipitated with ethanol. Bacteriophage vector M13 (mp18 and mp19) was digested with BamHI and SalI, extracted with phenol-chloroform, and precipitated with ethanol. The digested vector and PCR product were resuspended in water and ligated with T4 ligase (Boehringer Mannheim). Since BglII and BamHI produce compatible ends after digestion, the PCR products were cloned into phage M13 in known orientations. After ligation at room temperature for $4 \mathrm{~h}$, Escherichia coli JM101 cells were transfected with a plasmid (2). Single-stranded DNA from the bacteriophage (obtained from representative colorless plaques) was precipitated with polyethylene glycol, extracted with phenol-chloroform, and precipitated with ethanol. After suspension in distilled water, the single-stranded DNA was used as a template for DNA dideoxy chain termination sequencing in which Sequenase II (U.S. Biochemicals) and ${ }^{35}$ S-labeled dATP were used. The sequencing primers used to determine the sequence of the $N$. helminthoeca 16S rRNA gene included the universal M13 primers (Sequenase II kit; U.S. Biochemicals) and a set of internal $16 \mathrm{~S}$ rRNA primers which we designed (20). These primers are similar to those used by other workers (21). The gene was sequenced in both orientations by using the following primers: RA-17 (located at position 8 in Escherichia coli), 16 S-400 (located at position 371 in Escherichia coli; 5 '-CAATGGGCGAAAGC
CTGATCC-3'), 5003-18 (located at position 519 in Escherichia coli; 5'-GTAT TACCGCGGCTGCTG-3') 5004-20 (located at position 907 in Escherichia coli; 5'-CCGTCAATTCCTTTGAGTTT-3'), 2-20 (located at position 1181 in Escherichia coli; 5'-GACTTGACGTCATCCCCACC-3'), and R3-17 (located at position 1522 on the standard Escherichia coli 16S rRNA sequence).

Phylogenetic comparison. We performed phylogenetic analyses by using programs in the PHYLIP phylogenetic package (version 3.5) (5). A matrix of pairwise corrected nucleotide divergence values was constructed by using DNADIST and the Kimura two-parameter correction. This matrix was then used to produce a phylogenetic tree by the neighbor-joining method implemented in NEIGHBOR. The other sequences used to construct phylogenetic trees included the sequences of Ehrlichia sennetsu, Ehrlichia risticii, Ehrlichia phagocytophila, Ehrlichia equi, Ehrlichia bovis, Ehrlichia canis, Ehrlichia chaffeensis, Ehrlichia ewingii, Ehrlichia muris, Anaplasma marginale, Cowdria ruminantium, Wolbachia pipientis, Rickettsia prowazekii, Rickettsia rickettsii, Rickettsia bellii, an intracellular endosymbiont of Nasonia vitripennis, and the intracellular endosymbiont of Rhinocyllus conicus (20a)

Nucleotide sequence accession numbers. The GenBank accession number for the nucleotide sequence of the Escherichia coli 16S rRNA gene is J01695. The sequence of the 16S rRNA gene of $N$. helminthoeca has been deposited in the GenBank data library under accession number U12457. The accession numbers for the other sequences used to construct phylogenetic trees are as follows: Ehrlichia sennetsu, M73225; Ehrlichia risticii, M21290; Ehrlichia phagocytophila, M73220; Ehrlichia equi, M73223; Ehrlichia bovis, U03775; Ehrlichia canis, M73221; Ehrlichia chaffeensis, M73222; Ehrlichia ewingii, M73227; Ehrlichia muris, U15527; A. marginale, M60313; C. ruminantium, X61659; W. pipientis, X61768; Rickettsia prowazekii, M21789; Rickettsia rickettsii, U11021; Rickettsia bellii, U11014; an intracellular endosymbiont of Nasonia vitripennis, M84687; and the intracellular endosymbiont of Rhinocyllus conicus, M85267.

\section{RESULTS}

We obtained a 1,453-base sequence which corresponds approximately to bases 27 to 1478 in the complete rRNA gene sequence of Ehrlichia risticii (22). The sequence of the $N$. helminthoeca 16S rRNA gene was aligned with the 16S rRNA sequences of other members of the genus Ehrlichia, of members of the genus Rickettsia, and of representatives of several groups of insect-borne intracellular bacteria which have been placed in the alpha subgroup of the Proteobacteria. In agreement with the results of previous immunological studies, the $16 \mathrm{~S}$ rRNA gene sequence of $N$. helminthoeca is most similar to the sequences of Ehrlichia risticii and Ehrlichia sennetsu (levels of complete structural similarity [including insertion or deletion sites], 95.7 and $96.0 \%$, respectively). The results of a comparison performed with other representative intracellular bacteria belonging to the alpha subgroup of the Proteobacteria 


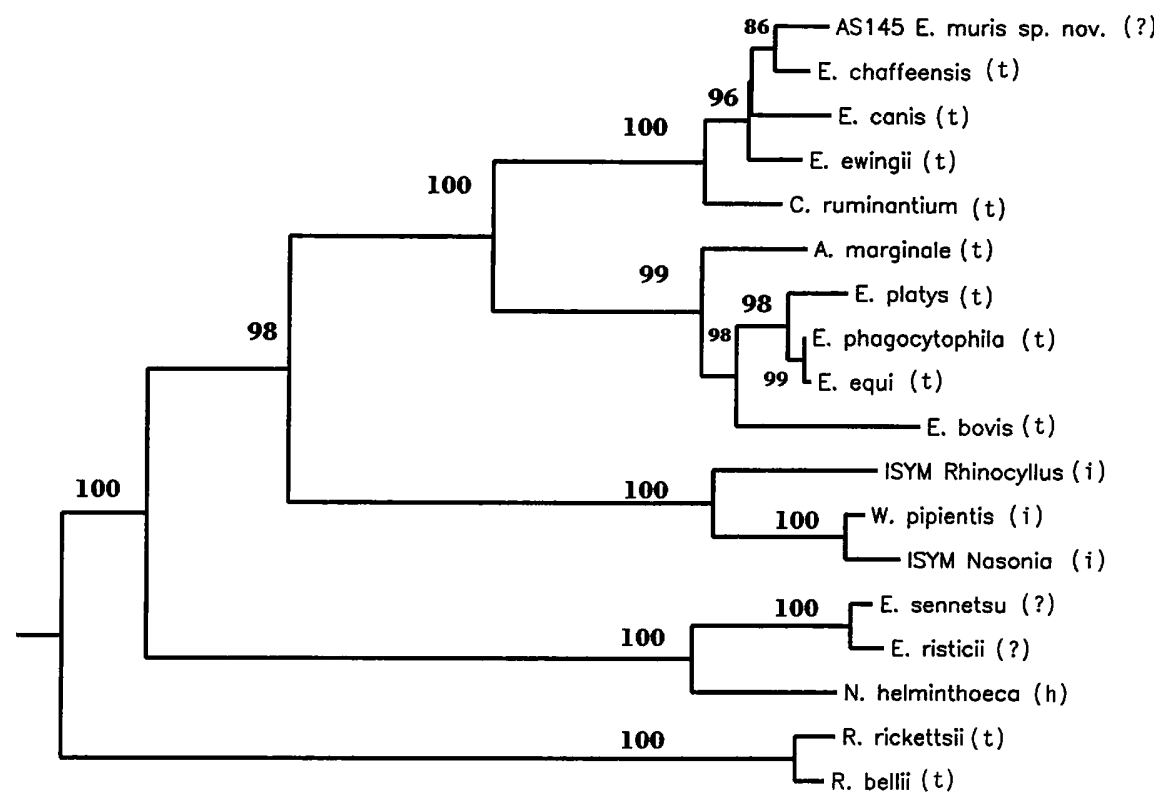

$1 \%$ Sequence divergence

FIG. 1. Phylogenetic relationships between N. helminthoeca and various intracellular bacteria belonging to the alpha subgroup of the Proteobacteria. The tree was constructed by using a neighbor-joining method (NEIGHBOR in PHYLIP) from a matrix of corrected nucleotide divergence values. The root of the tree was determined by using the sequences of Rochalimaea quintana and Agrobacterium tumefaciens as outliers. The evolutionary distance (percentage of nucleotide divergence) between a pair of species was calculated by adding the distances along the horizontal axis from the last common ancestor of the terminal taxa. The numbers on the branches are the bootstrap values calculated in PHYLIP; only bootstrap values greater than 50\% are shown. Modes of transmission are indicated in parentheses, as follows: (h), helminth; (i), insect; (t), tick; (?), unknown. ISYM Rhinocyllus, incompatibility intracellular symbiont of Rhinocyllus sp.; ISYM Nasonia, incompatibility intracellular symbiont of Nasonia sp.

are shown in Table 1. Our phylogenetic analysis of $16 \mathrm{~S}$ rRNA sequences included more taxa, including all available sequences for members of the genus Ehrlichia and the insect intracellular endosymbionts which are phylogenetically related to the genus Ehrlichia. The data in Table 1 include data for only a representative sample of these taxa. The most closely related Ehrlichia species other than members of the $N$. helminthoeca-Ehrlichia risticii-Ehrlichia sennetsu group was Ehrlichia equi, which exhibited only $86.4 \%$ 16S rRNA sequence similarity to $N$. helminthoeca.

Figure 1 shows the phylogenetic relationships among various taxa, as determined by the neighbor-joining method. The genus Ehrlichia is divided into three well-differentiated groups. The cluster which includes $N$. helminthoeca, Ehrlichia risticii, and Ehrlichia sennetsu is a highly distinct clade, and the average level of corrected sequence difference from all other species belonging to the genus Ehrlichia is 16 to $17 \%$. This clade is only slightly more closely related to the other Ehrlichia clusters than it is to the members of the genus Rickettsia (average distance, $19.3 \%$ ). It represents the deepest branch within the tribe Ehrlichieae.

Each of the three clades of the genus Ehrlichia is characterized by being associated with a bacterium that is not classified as an Ehrlichia species. In addition to the $N$. helminthoeca triad, C. ruminantium clusters with three Ehrlichia species, including Ehrlichia canis, while in the third cluster $A$. marginale clusters with four members of the genus Ehrlichia. Thus, the three Ehrlichia clusters are separated from each other by at least two noncongeneric named forms. The average level of intercluster sequence divergence between members of the Cowdria- and Anaplasma-associated clusters is about $7.5 \%$. In addition, there appears to be some similarity in the invertebrate vectors for the Ehrlichia species which cluster with mem- bers of the genera Cowdria and Anaplasma. In all cases in which a vector is known, the species are associated with ticks. The vectors for Ehrlichia risticii and Ehrlichia sennetsu have not been identified, despite searches for an arthropod vector.

An additional sister clade of the tick-borne Ehrlichia clade is shown in Fig. 1. There is a significant cluster of insect-borne intracellular endosymbionts, which includes $W$. pipientis. The members of this clade are more similar to the tick-borne members of the genus Ehrlichia than either they or the tick-borne ehrlichial species are to the members of the $N$. helminthoeca cluster. The average level of sequence divergence between the insect endosymbionts and the tick-borne members of the genus Ehrlichia is $14.2 \%$, while the average level of divergence between the insect endosymbionts and members of the $N$. helminthoeca cluster is $17.4 \%$. The inclusion of the insect-borne intracellular endosymbionts with the tick-borne members of the genus Ehrlichia creates a distinct group of sister clades which are significantly differentiated both from the helminthborne Neorickettsia species and the associated Ehrlichia species and from the tick-borne Rickettsia species. We propose that the $N$. helminthoeca cluster should be referred to as the "neoehrlichia" cluster.

\section{DISCUSSION}

The causative agent of salmon poisoning disease (10), $N$. helminthoeca, was described by Philip in 1953. On the basis of disease and morphological characteristics, early investigators were not able to place the organism in a previously described genus, and the genus Neorickettsia is still classified as an independent group within the tribe Ehrlichieae $(15,18)$. The species $N$. helminthoeca is unique in its association with a helminth vector. Our data suggest that there is a strong and unique 
phylogenetic association among three intracellular bacterial species, N. helminthoeca, Ehrlichia risticii, and Ehrlichia sennetsu. The results of a phylogenetic analysis indicate that these organisms have been differentiated from other previously studied intracellular members of the alpha subgroup of the Proteobacteria for a very long time. Ehrlichia risticii is the agent of Potomac horse fever, an acute diarrheic disease of equids whose nonmammalian vector and mode of transmission remain unknown (19). Ehrlichia sennetsu, the causative agent of Sennetsu fever in Japan, is anecdotally associated with the consumption of a particular raw fish (6).

Ultrastructurally, $N$. helminthoeca is similar to other ehrlichial species. All of these organisms are intracellular bacteria with two unit membranes, ribosomes, and fine DNA strands. However, $N$. helminthoeca organisms are tightly enveloped by the host membrane, like Ehrlichia sennetsu and Ehrlichia risticil but unlike Ehrlichia canis, which produces morula inclusions containing large numbers of organisms (16). N. helminthoeca, Ehrlichia risticii, and Ehrlichia sennetsu infect monocytes and macrophages rather than granulocytes. However, during infection none of these species produces large dense morulae, in contrast to other members of the genus Ehrlichia, such as Ehrlichia canis. The antigenic cross-reactivity between $N$. helminthoeca and several members of the genus Ehrlichia has been investigated previously (14). The results of indirect fluorescent labeling and Western blot (immunoblot) experiments make it clear that Ehrlichia sennetsu and Ehrlichia risticii are antigenically more similar to $N$. helminthoeca than to Ehrlichia canis, Ehrlichia equi (14), or ehrlichial strain AS145, which was isolated from a wild mouse in Japan (8).

The results of a comparison of the sequences of the $16 \mathrm{~S}$ rRNA genes of various intracellular bacteria, including members of the genus Rickettsia and other members of the genus Ehrlichia, confirmed that $N$. helminthoeca shares a close common ancestry with Ehrlichia risticii and Ehrlichia sennetsu. In addition, since the results of a phylogenetic analysis indicated that this trio of species is not closely related to any of the arthropod-borne Rickettsia or Ehrlichia species or to the $W$. pipientis-like insect endosymbionts, it is not unreasonable to hypothesize that the three species may be transmitted by a different vector, such as the fluke vector which is known to be involved in the $N$. helminthoeca life cycle.

The possibility that $N$. helminthoeca, Ehrlichia risticii, and Ehrlichia sennetsu are all helminth-associated bacteria can now be examined by using the 16S rRNA gene as a molecular probe. The evidence that $N$. helminthoeca is present in various stages of the life cycle of its trematode vector is based only on the results of transmission experiments performed with dogs. Our data suggest that the $16 \mathrm{~S}$ rRNA sequence could be used as a probe to determine the infection rates of each stage of the trematode life cycle and to diagnose salmon poisoning disease in dogs. Furthermore, the search for vectors of Potomac horse fever and Sennetsu fever should be broadened to include possible infection of fish or snail populations in geographic foci of infection by trematodes which could act as the vectors of ehrlichial infection.

Using the phylogenetic evidence presented above, we suggest that the close clustering of the three species $N$. helminthoeca, Ehrlichia risticii, and Ehrlichia sennetsu should eventually result in careful reconsideration of nomenclature of the genus Ehrlichia. The inclusion of many intracellular bacteria, including members of the genera Cowdria and Anaplasma and a number of Wolbachia sp. strains (data not shown), in the broad cluster represented by the named Ehrlichia species weakens the coherence of the taxon Ehrlichia as a genus. The genetic differences between the Neorickettsia cluster and the two other clusters of Ehrlichia species are almost equivalent to the genetic differences between any of the Ehrlichia or Wolbachia species and Rickettsia species. Thus, data from the phylogenetic analysis, together with other biological characteristics, suggest that Ehrlichia risticii and Ehrlichia sennetsu should eventually be removed from the genus Ehrlichia and reclassified together with $N$. helminthoeca. In addition, the results of our analysis strongly suggest that consideration should be given to reclassification of $A$. marginale and $C$. ruminantium together with associated members of the genus Ehrlichia.

Finally, it is worth noting that the diversity of forms in the phylogenetic cluster which contains $N$. helminthoeca is likely to be greater in the future. Additional studies of the mode of transmission and the phylogenetic placement of the two other members of the genus Neorickettsia, the elusive organism $\mathrm{Ne}$ orickettsia elokominica (4) and the SF agent (6), in relation to $N$. helminthoeca and to other Ehrlichia species are under way.

\section{ACKNOWLEDGMENTS}

We thank Jim Laird and Holly Ferrell for culturing and purifying neorickettsial organisms and DNA.

This study was supported in part by grant RO1AI30010 from the National Institutes of Health and by a canine research grant from The Ohio State University.

\section{REFERENCES}

1. Anderson, B., J. Dawson, D. Jones, and K. Wilson. 1991. Ehrlichia chaffeensis, a new species associated with human ehrlichiosis. J. Clin. Microbiol. 29:2838-2842.

2. Ausubel, F. M., R. Brent, R. E. Kingston, D. D. Moore, J. G. Seldman, J. A. Smith, and K. Struhl. 1987. Current protocols in molecular biology. John Wiley and Sons, New York.

3. Cordy, D. R., and J. R. Gorham. 1950. The pathology and etiology of salmon disease in dog and fox. Am. J. Pathol. 26:617-637.

4. Farrell, R. K. 1964. Transmission of two rickettsia-like disease agents of dogs by endoparasites in northwestern U.S.A., abstr. 0162BIO, p. 438-439. In A. Corradetti (ed.), Program and abstracts of the 1st International Congress of Parasitology. Pergamon Press, Oxford.

5. Felsenstein, J. 1989. Phylogeny inference package (version 3.3). Cladistics 5:164-166.

6. Fukuda, T., T. Sasahara, and T. Kitao. 1973. Studies on the causative agent of Hyuganetsu disease. XI. Characteristics of rickettsia-like organism isolated from metacercaria of Stellantchamus falcatus parasitic in grey mullet. J. Jpn. Assoc. Infect. Dis. 47:474-482. (In Japanese.)

7. Gorham, J. R., and W. J. Foreyt. 1984. Salmon poisoning disease, p. 538-544. In C. E. Greene (ed.), Clinical microbiology and infectious diseases of the dog and cat. W. B. Saunders Co., Philadelphia.

8. Kawahara, M., C. Suto, Y. Rikihisa, S. Yamamoto, and Y. Tsuboi. 1993. Characterization of ehrlichial organisms isolated from a wild mouse. J. Clin. Microbiol. 31:89-96.

9. Knapp, S. E., and R. E. Millemann. 1981. Salmon poisoning disease, p. 376-387. In J. Davis (ed.), Infectious diseases of wild mammals. Iowa State University Press, Ames.

10. Philip, C. B. 1953 . Nomenclature of the rickettsiaceae pathogenic to vertebrates. Ann. N. Y. Acad. Sci. 56:484-494.

11. Philip, C. B., W. J. Hadlow, and L. E. Hughes. 1953. Neorickettsia helmintho$e c a$, a new rickettsia-like disease agent of dogs in western United States transmitted by a helminth, p. 256-257. In International Congress on Microbiology report: proceedings of the 6th congress, vol. 2. International Association of Microbiological Societies, Rome.

12. Philip, C. B., W. J. Hadlow, and L. E. Hughes. 1954. Studies on salmon poisoning disease in canines. I. The rickettsial relationships and pathogenicity of Neorickettsia helminthoeca. Exp. Parasitol. 3:336-350.

13. Pretzman, C. I., Y. Rikihisa, D. Ralph, J. C. Gordon, and S. Bech-Nielson. 1987. Enzyme-linked immunosorbent assay for Potomac horse fever disease. J. Clin. Microbiol. 25:31-36.

14. Rikihisa, Y. 1991. Cross-reacting antigens between Neorickettsia helminthoeca and Ehrlichia species, shown by immunofluorescence and Western immunoblotting. J. Clin. Microbiol. 29:2024-2029.

15. Rikihisa, Y. 1991. The tribe Ehrlichieae and ehrlichial diseases. Clin. Microbiol. Rev. 4:286-308.

16. Rikihisa, Y., S. A. Ewing, J. C. Fox, A. G. Siregar, F. H. Pararibu, and M. B. Malole. 1992. Analysis of Ehrlichia canis and a canine granulocytic Ehrlichia infection. J. Clin. Microbiol. 30:143-148.

17. Rikihisa, Y., H. Stills, and G. Zimmerman. 1991. Isolation and continuous 
culture of Neorickettsia helminthoeca in a macrophage cell line. J. Clin. Microbiol. 29:1928-1933.

18. Ristic, M., and D. Huxall. 1984. Tribe II. Ehrlichieae, p. 704-711. In N. R. Krieg and J. G. Holt (ed.), Bergey's manual of systematic bacteriology, vol 1. The Williams \& Wilkins Co., Baltimore.

19. Schmidmann, E. T., R. M. Rice, M. G. Rohle, and A. F. Azad. 1988. Search for an arthropod vector of Ehrlichia risticii, p. 9-16. In Proceedings of a symposium on Potomac horse fever. Veterinary Learning Systems, Lawrenceville, N.J.
20. Stothard, D. S J. A. Clark, and P. A. Fuerst. 1994. The ancestral divergence of Rickettsia bellii from the spotted fever and typhus groups of Rickettsia, and the antiquity of the genus Rickettsia. Int. J. Syst. Bacteriol. 44:798-804.

20a.Turner, C., B. C. Campbell, and T. S. Bragg. Unpublished data.

21. Weisburg, W., S. Barnes, D. Pelletier, and D. Lane. 1991. 16S ribosomal DNA amplification for phylogenetic study. J. Bacteriol. 173:697-703.

22. Weisburg, W., M. Dobson, J. Samuel, G. Dasch, L. Mallavia, O. Baca, L Mandelco, J. Sechrest, E. Weiss, and C. Woese. 1989. Phylogenetic diversity of the rickettsiae. J. Bacteriol. 171:4202-4206. 